\title{
Severe acute respiratory syndrome coronavirus 2 (SARS-CoV2) in Kidney transplant patients: A case series and literature review
}

\author{
Muzamil Latief ${ }^{1}$, Obeid Shafi ${ }^{2}$, Zhahid Hassan ${ }^{3}$, Summyia Farooq ${ }^{4}$, Farhat Abbas ${ }^{* 4}$ (ID \\ Received: 10 Aug 2020 \\ Published: 3 Feb 2021
}

\begin{abstract}
COVID-19 is a novel highly contagious disease caused by the severe acute respiratory syndrome coronavirus 2 (SARS-CoV-2). Immunosuppressed people are at a higher risk for unfavourable outcomes if infected with SARS-CoV-2, as cellular immunity plays a key role in determining the course and outcome in COVID-19. Kidney transplant recipients (KTRs) are thus a distinct subset of the population. We describe our early experience with 2 KTRs requiring hospital admission due to COVID-19 and who recovered well. We conclude that timely intervention in the form of modifying immunosuppression and close monitoring and institution of further measures based on clinical severity is needed in KTRs with COVID-19.
\end{abstract}

Keywords: Severe acute respiratory syndrome coronavirus 2, Kidney transplant

\author{
Conflicts of Interest: None declared \\ Funding: None \\ *This work has been published under CC BY-NC-SA 1.0 license. \\ Copyright $₫$ Iran University of Medical Sciences
}

Cite this article as: Latief M, Shafi O, Hassan Z, Farooq S, Abbas F. Severe acute respiratory syndrome coronavirus 2 (SARS-CoV2) in Kidney transplant patients: A case series and literature review. Med J Islam Repub Iran. 2021 (3 Feb);35:17. https://doi.org/10.47176/mjiri.35.17

\section{Introduction}

COVID-19 is a novel highly contagious disease caused by the severe acute respiratory syndrome coronavirus 2 (SARS-CoV-2), a recently discovered RNA $\beta$ - coronavirus $(1,2)$. The first case was reported in China in December 2019, and in the span of a few months, the virus has spread to over 180 countries and infected over 1 crore people resulting in the death of over 5 lakh people (3). The first case in India was confirmed on 30 Jan 2020, and the rate of infection has increased at a significant rate in the last couple of months. India faces a unique challenge from SARS-CoV-2 given its characteristics like dense population, poor hand hygiene practices, high prevalence of chronic illnesses like diabetes mellitus (DM) and hypertension, and the limited health infrastructure and resources (4). Initially, the rate of increase in the number of cases for India was relatively subdued compared to the USA and Europe, primarily due to the strict lockdown enforced by the government, giving the health system valuable time to prepare for the onslaught. The number of cases has seen a spurt in the last couple of months, threatening to overwhelm the health care resources.

Immunosuppressed people are at a higher risk for unfa- vourable outcomes if infected with SARS-CoV-2, as cellular immunity plays a key role in determining the course and outcome in COVID-19 (5). Kidney transplant recipients (KTRs) are thus a distinct subset of the population, and in the absence of evidence from large robust clinical trials, the management of COVID-19 is guided mainly by early case reports and experience with managing other infections in KTR patients.

We describe our early experience with 2 KTRs requiring hospital admission due to COVID-19 and review current recommendations and best practice guidelines.

\section{Case series}

Case 1

A 30-year-old male with a history of live related renal transplant (LRRT) 3 years back, who was doing well on triple immunosuppression, presented with low grade fever and throat pain for 5 days with 2 days of myalgias. There was no history of cough, haemoptysis, breathlessness, chest discomfort, vomiting, dysuria, or abdominal pain. Pulse rate was 104 per minute, and BP was 130/80. Systemic examination was unremarkable except for a subcen-

\footnotetext{
Corresponding author: Dr Farhat Abbas, farahabbas.m@gmail.com

1. Nephrology Division, GMC Secunderabad, Hyderabad

2. Flushing Hospital, Medical Center, New York, USA

3. Consultant Medicine/Endocrinology, GMC, Baramulla, Kashmir, India

4. Pathology Division, GMC, Srinagar, Kashmir
} 
trimetric submandibular lymph nodes. Temperature was 100.2 Fahrenheit. Laboratory evaluation revealed total leucocyte count 6600/microliter with lymphopenia (12\%) with a normal haemoglobin; cultures were sterile and the result of liver function test was normal. Urine examination result was normal. The serum creatinine was $0.8 \mathrm{mg} / \mathrm{dL}$, with normal $\mathrm{x}$-ray chest and ultrasound abdomen and graft. Cytomegalovirus (CMV) and BK viral markers were negative. C-reactive protein was elevated $(12 \mathrm{mg} / \mathrm{l})$. RT PCR for SARS-CoV2 turned out to be positive. The patient was put on azithromycin, oseltamivir and continued on the same dose of tacrolimus $1 \mathrm{mg} \mathrm{BD}$, and prednisolone dose increased from $5 \mathrm{mg}$ to $20 \mathrm{mg}$ OD, while the dose of mycophenolate mofetil halved from $1000 \mathrm{mg}$ to $500 \mathrm{mg}$ daily in 2 divided doses. The patient became afebrile on day 3 of hospitalization. Lymphopenia improved completely by day 9 of hospital stay. Repeat RT PCR was negative on day 17 . Renal functions remained stable throughout the hospital stay and the patient was discharged on the same immunosuppression as was commenced in hospital; and after a total of 4 weeks, his immunosuppression was back to preinfection dose and the patient was doing well.

\section{Case 2}

A 35 year-old male, with hypertension, post-LRRT on triple immunosuppression, and telmisartan 40mg daily, and 5 years posttransplant, was admitted with high grade fever for 5 days and few episodes of vomiting since morning. BP was 104/70 with tachycardia (Pulse: 110/minute). The patient received IV fluid resuscitation on arrival and his antihypertensive medication was withheld. Viral serology for CMV, BKV was negative and the result of urine examination was normal. His last serum creatinine was $0.9 \mathrm{mg} / \mathrm{dL}$ ( 2 months ago) and it was $2.3 \mathrm{mg} / \mathrm{dL}$ during hospitalization. There was lymphopenia (14\% lymphocytes) with normal haemoglobin of $12 \mathrm{~g} / \mathrm{dL}$. There was a 3-fold increase in AST and ALT with normal bilirubin in liver function test. X-ray chest and abdominal ultrasound and graft was normal. Blood and urine cultures were sterile. RT PCR from nasopharyngeal swab was positive for SARS-CoV2. The patient was continued on tacrolimus $1 \mathrm{mg}$ (morning) and $0.5 \mathrm{mg}$ (evening). Prednisolone was increased from $5 \mathrm{mg}$ OD to $20 \mathrm{mg}$ OD.

Mycophenolate sodium was stopped. Oral azithromycin, along with hydroxychloroquine and oseltamivir, was given and haemodynamic and fluid status were closely monitored. The patient became afebrile on day 4 and serum creatinine gradually decreased to $1.1 \mathrm{mg} / \mathrm{dL}$ on day 15 . RT PCR was negative on day 21 . He was given antihypertensive medication again and continued the dual immunosuppression at hospital discharge. On follow-up (telephonic), the patient was doing well. His tacrolimus level was $7 \mathrm{ng} / \mathrm{mL}$ and leukopenia had completely settled. The patient was resumed on triple immunosuppression with the same dose of tacrolimus, prednisolone $10 \mathrm{mg}$, and mycophenolate sodium $360 \mathrm{mg}$, BD, and is doing well with the latest creatinine of $1 \mathrm{mg} / \mathrm{dL}$.

\section{Discussion}

Immunosuppressive therapy forms the cornerstone of management for posttransplant patients with the goal of preventing rejection of the graft. Induction therapy, administered at or around the time of transplantation to prevent acute rejection, usually consists of biologic antibodies (rabbit antithymocyte globulin, basiliximab) and highdose glucocorticoids. Maintenance therapy, where the degree of immunosuppression is usually less than initiation therapy, is generally lifelong. The regimen can include glucocorticoids, calcineurin inhibitors (CNIs; tacrolimus or cyclosporine), antimetabolic agents (mycophenolate mofetil or azathioprine), mammalian (mechanistic) target of rapamycin (mTOR) inhibitors (sirolimus or everolimus), and orcostimulatory blockade agents (belatacept) (6). These patients require close follow-up as a result of complex immunosuppression, which makes them susceptible to malignancy and infection, in addition to the underlying multiple comorbidities that usually exist in such patients.

Viral infections like respiratory syncytial virus (RSV), adenovirus, and influenza have all been documented to cause more severe illness in transplant patients (7-10). COVID-19 has similarly been linked to more severe illness and more rapid progress in immunocompromised hosts, resulting in increased intensive care unit (ICU) admissions and mortality (11). Most studies in KTRs with COVID-19 reported mortalities between $23 \%$ to $28 \%$, which is much higher than the mortality reported in the general population $(\leq 5 \%)(12-14)$. The host response to SARS-CoV-2 plays an important role in disease presentation and severity. Lymphopenia, which is a laboratory feature associated with severe disease in COVID-19, is exacerbated by the use of immunosuppressive drugs, which possess the inherent potential to decrease the number of lymphocytes and/or impair their function (5). Fever may be less common at presentation in KTRs compared to the general population $(11,12)$. Also, a hyperimmune response is seen in critically ill patients with COVID-19, reflecting the delicate balance between protective and dysregulated host response (5). There are also concerns that COVID-19 itself may increase the risk of acute rejection. The recommendations for the prevention of COVID19 in the general population hold true for KTR patients, which include strict hygiene measures like frequent handwashing, wearing masks in crowded places, and physical distancing (15). Elective surgical procedures including living-related kidney transplants had been stopped initially in India as per the government recommendations, although the restrictions have since been lifted. However, performing renal transplant at the time of the COVID-19 pandemic should be done with an abundance of caution and at centers where facilities for management of COVID19 patients are available (16). Very limited data regarding immunosuppression at the time of induction is available as most centers worldwide have refrained from performing elective transplants. We suggest that altering the immunosuppression in the first 3 to 6 months posttransplant should be taken on a case-by-case basis, as the risk for graft rejection is highest. Also, basiliximab may be pre- 
ferred over rATG and alemtuzumab for induction treatment in kidney transplantation, as it causes less lymphocyte depletion (5). Asymptomatic to mild cases can potentially be managed as outpatients, maintaining close follow-up via telemedicine (15). This aspect might become more relevant in our scenario in the coming weeks to months as the number of cases rise in India, causing a crunch for our health care system. However, this approach should be individualized given that it involves a remote assessment, taking into account insensitive subjective information like worsening of dyspnea on exertion and/or self-reported vital signs. Furthermore, there is the potential for rapid clinical decompensation in COVID-19, as reported in patients who eventually develop acute respiratory distress (13).

There is a paucity of robust clinical studies; thus, guidelines regarding the management of immunosuppression in KTR patients could be critical to managing these patients (15). However, in vitro studies have suggested that coronavirus requires intact immunophilin pathways, which when inhibited by cyclosporine could affect the growth of SARS-CoV-2, although the clinical significance of these observations is not known $(17,18)$. In addition, complete withdrawal of immunosuppression could potentially worsen the hyperimmune response seen in critically ill patients (7). Also, experimental data suggest that certain immunosuppressive agents such as mTOR inhibitors may have some biological activity against SARS-CoV-2 (19). Thus, the modification of the immunosuppressive regimen in KTR with COVID-19 should be preceded by a review of the patient history, accounting for age and comorbidities, the time since transplant, and the severity of COVID-19 infection. Most studies reported discontinuation of antiproliferative agents at COVID-19 diagnosis or admission to hospital $(11,13,14,20)$. Akalin et al and Banerjee et al in their studies reported withholding of CNIs in severe disease, while the Columbia University KT group continued tacrolimus with target trough levels of 4 to $7 \mathrm{ng} / \mathrm{mL}$ (13). Steroids were continued in most reviews at the same or higher doses $(13,14)$. Lubetzy et al in their unpublished retrospective study of $54 \mathrm{KTRs}$ have reported the lowest in-hospital case fatality rate (8\%) from 39 inpatients (21). They discontinued antimetabolites in $61 \%$ of hospitalized patients while tacrolimus was continued (with a decrease in dose in $46 \%$ of inpatients). This suggests that reduction of immunosuppression could also be a reasonable approach instead of complete cessation. We suggest discontinuation of antiproliferative agents on admission to the hospital in KTR patients with COVID-19. Steroids should be continued at the same or increased doses as has been practiced at most centers. CNIs can be continued in mild to moderate cases at the same or reduced doses, but should be discontinued in severe COVID-19 requiring ICU admission. A European working group suggests the use of cyclosporine preferably over tacrolimus in view of its potential specific antiviral properties and its use in combination with MMF (when needed) for its favourable pharmacokinetics (5). A trial for anti-IL-6 agents could be given in critical illness with evidence of cytokine storm. Also, plasmapheresis or IVIG should be considered as nonim- munosuppressive/immunomodulatory strategies, with the use of convalescent plasma during plasmapheresis. Immunosuppressive drugs should be cautiously reintroduced after signs of recovery, generally after 5 to 15 days of symptoms resolution (5). There are no approved COVID19 specific therapies at the time of writing this review, although various potential agents have been identified and RCTs are ongoing. It is important to be vigilant and prudent regarding the possible interactions between these agents and the immunosuppressive regimen in KTRs. Remdesivir and dexamethasone are the most promising treatments at this time $(22,23)$. Remdesivir, a novel nucleotide analog, has shown some clinical benefits in trials, decreasing the time to recovery from severe COVID-19. It is administered intravenously, has a favourable clinical profile, and no known interactions. However, its use has not been studied in patients with compromised renal function and its use is not recommended in patients with eGFR less than $30 \mathrm{~mL}$ per minute $(24,25)$. Dexamethasone was used via the oral or intravenous route in an open-label large randomized trial and showed a reduction in 28-day mortality in hospitalized patients in a preliminary report (26). Other therapies that are being studied are hydroxychloroquine (HCQ)/chloroquine with or without azithromycin, lopinavir-ritonavir (LPV/r), favipiravir, tocilizumab, and convalescent plasma. They are recommended to be used only in the context of a clinical trial $(27,28)$. Caution should be exercised with the concomitant use of HCQ or LPV/r with tacrolimus, as toxicity from elevated levels has been reported (29). Our experience is an early one but was reassuring probably because of timely alteration of immunosuppression and close clinical monitoring of patients.

\section{Conclusion}

A high index of suspicion of SARS-CoV2 infection in vulnerable patient population like KTRs cannot be overemphasized. Timely intervention in the form of modifying immunosuppression and close monitoring and institution of further measures based on clinical severity is needed. As the pandemic is here to stay, more and more studies and therapeutic agents will come forth and help save precious lives.

\section{Conflict of Interests}

The authors declare that they have no competing interests.

\section{References}

1. Wu F, Zhao S, Yu B, Chen YM, Wang W, Song ZG, et al. A new coronavirus associated with human respiratory disease in China. Nature. 2020;579:265-269.

2. World Health Organization. Director-General's remarks at the media briefing on 2019-nCoV on 11 February 2020. Available at: https://www.who.int/dg/speeches/detail/who-director-general-s-rema rks-at-the-media-briefing-on-2019-ncov-on-11-february-2020. Access ed June14, 2020.

3. Johns Hopkins University of Medicine-Coronavirus Resource Center. Coronavirus COVID-19 global cases by the Center for Systems Science and Engineering (CSSE) at Johns Hopkins University. June 29, 2020. Available at: https://coronavirus.jhu.edu/map.html. Accessed June 29, 2020. 
4. Kamath S, Kamath R. Salins PCOVID-19 pandemic in India: challenges and silver linings. Postgrad Med J. 2020;96:422-423.

5. Andreas Kronbichler, Philipp Gauckler, Martin Windpessl, Jae Il Shin, Vivekanand Jha, Brad H. Rovin et al COVID-19: implications for immunosuppression in kidney disease and transplantation. Nat Rev Nephrol. 2020;16:365-367.

6. Voora S, Adey DB. Management of Kidney Transplant Recipients by General Nephrologists: Core Curriculum 2019. Am J Kidney Dis. 2019;73(6):866-879

7. Maggiore U, Abramowicz D, Crespo M, Mariat C, Mjoen G, Peruzzi $\mathrm{L}$, et al. How should I manage immunosuppression in a kidney transplant patient with COVID-19? An ERA-EDTA DESCARTES expert opinion. Nephrol Dial Transplant. 2020;35(6):899-904.

8. Patel R, Paya CV. Infections in solid-organ transplant recipients. Clin Microbiol Rev. 1997;10(1):86-124.

9. Razonable RR. Management of viral infections in solid organ transplant recipients. Expert Rev Anti Infect Ther. 2011;9(6):685-700.

10. Manuel O, López-Medrano F, Keiser L, Welte T, Carratalà J, Cordero E, et al. Influenza and other respiratory virus infections in solid organ transplant recipients. Clin Microbiol Infect. 2014;20 Suppl 7(Suppl 7):102-108

11. Akalin E, Azzi Y, Bartash R, Seethamraju H, Parides M, Hemmige $\mathrm{V}$, et al. Covid-19 and Kidney Transplantation. N Engl J Med. 2020;382(25):2475-2477.

12. Pereira MR, Mohan S, Cohen DJ, Husain SA, Dube GK, Ratner LE, et al. COVID-19 in solid organ transplant recipients: Initial report from the US epicenter [published online ahead of print, 2020 Apr 24]. Am J Transplant. 2020;10.1111/ajt.15941.

13. Columbia University Kidney Transplant Program. Early Description of Coronavirus 2019 Disease in Kidney Transplant Recipients in New York. J Am Soc Nephrol. 2020;31(6):1150-1156.

14. Alberici F, Delbarba E, Manenti C, Econimo L, Valerio F, Pola A, et al. A single center observational study of the clinical characteristics and short-term outcome of 20 kidney transplant patients admitted for SARS-CoV2 pneumonia. Kidney Int. 2020;97(6):1083-1088.

15. Gleeson SE, Formica RN, Marin EP. Outpatient Management of the Kidney Transplant Recipient during the SARS-CoV-2 Virus Pandemic [published online ahead of print, 2020 Apr 28]. Clin J Am Soc Nephrol. 2020;15(6):892-895.

16. Kute V, Varugese S, Prasad N, Shroff S, Agarwal SK. On behalf of COVID-19 Working Group of Indian Society of Nephrology. Renal transplant guidelines with reference to COVID-19 infection. Indian J Nephrol. 2020;30:176-8.

17. Carbajo-Lozoya J, Müller MA, Kallies S, Thiel V, Drosten C, von Brunn A. Replication of human coronaviruses SARS-CoV, HCoVNL63 and HCoV-229E is inhibited by the drug FK506. Virus Res. 2012;165(1):112-117.

18. Ma-Lauer Y, Zheng Y, Malešević M, von Brunn B, Fischer G, von Brunn A. Influences of cyclosporin $\mathrm{A}$ and non-immunosuppressive derivatives on cellular cyclophilins and viral nucleocapsid protein during human coronavirus 229E replication. Antiviral Res. 2020;173:104620.

19. Conti P, Ronconi G, Caraffa A, Gallenga CE, Ross R, Frydas I, et al. Induction of pro-inflammatory cytokines (IL-1 and IL-6) and lung inflammation by Coronavirus-19 (COVI-19 or SARS-CoV-2): antiinflammatory strategies [published online ahead of print, $2020 \mathrm{Mar}$ 14]. J Biol Regul Homeost Agents. 2020;34(2):1.

20. Banerjee D, Popoola J, Shah S, Ster IC, Quan V, Phanish M. COVID-19 infection in kidney transplant recipients. Kidney Int 2020;97(6):1076-1082.

21. Lubetzky M, Aull M, Craig-Schapiro R, Lee JB, Lee JR, Sultan S et al. Kidney Allograft Recipients Diagnosed with Coronavirus Disease-2019: A Single Center Report. doi:10.1101/2020.04.30.20086462. Available at: https://www. medrxiv.org/content/10.1101/2020.04.30.20086462v2. Accessed June 25,2020 .

22. Beigel JH, Tomashek KM, Dodd LE, Mehta AK, Zingman BS, Kalil AC, et al. Remdesivir for the Treatment of Covid-19 -Preliminary Report [published online ahead of print, 2020 May 22]. N Engl J Med. 2020; NEJMoa2007764.

23. Wang Y, Zhang D, Du G, Du R, Zhao J, Jin Y, et al. Remdesivir in adults with severe COVID-19: a randomised, double-blind, placebocontrolled, multicentre trial [published correction appears in Lancet. 2020 May 30;395(10238):1694]. Lancet. 2020;395(10236):1569-1578.

24. Grein J, Ohmagari N, Shin D, Diaz G, Asperges E, Castagna A, et al.
Compassionate Use of Remdesivir for Patients with Severe Covid-19. N Engl J Med. 2020;382(24):2327-2336.

25. US Food and Drug Administration: Fact Sheet for Health Care Providers Emergency Use Authorization (EUA) of Remdesivir (GS5734 ${ }^{\mathrm{TM}}$ ), Silver Spring, MD, US Food and Drug Administration, 2020. Available at: https://www.fda.gov/media/137566/download. Accessed June 28, 2020.

26. RECOVERY Collaborative Group. Effect of Dexamethasone in Hospitalized Patients with COVID-19 - Preliminary Report. Available at: https://www.medrxiv.org/content/10.1101/2020.06.22.201372 73v1 full.pdf . Accessed on June 25, 2020.

27. National Institutes of Health. Coronavirus Disease 2019 (COVID-19) Treatment Guidelines. Available at: https:/ covid19treatmentguidelines.nih.gov. Accessed on June 26, 2020.

28. Infectious Diseases Society of America Guidelines on the Treatment and Management of Patients with COVID-19. Available at: https://www.idsociety.org/practice-guideline/covid-19-guidelinetreatment-and-managem ent/. Accessed on: June 26, 2020.

29. Xia T, Wang Y. Coronavirus disease 2019 and transplantation: The combination of lopinavir/ritonavir and hydroxychloroquine is responsible for excessive tacrolimus trough level and unfavorable outcome. Am J Transplant. 2020. doi:10.1111/ajt.15992. 